\title{
An Orthogonal Collocation Method for Sobolev Equations Xiaofei $\mathrm{Lu}^{1, \mathrm{a}}$ and Ning $\mathrm{Ma}^{2, \mathrm{~b}^{*}}$ \\ ${ }^{1}$ China University of Petroleum, Beijing, China \\ ${ }^{2}$ China University of Petroleum, Beijing, China \\ aluxf012@126.com, bbjmaning@163.com;
}

Keywords: sobolev equations; orthogonal collocation method; error estimate.

Abstract. In this article, an orthogonal collocation method for the initial-boundary value problem for sobolev equations is given. Optimal order estimates are obtained for the approximation of the method.

\section{Introduction}

Sobolev equation appears in many areas of physics. The study about sobolev equations has important significance for solving physical engineering problems. With the sobolev equations we can analyze the flow of fluids through fissured rock, and obtain the process of the migration of the moisture in soil and solve thermodynamics problems, etc. There have been many numerical modeling methods for sobolev equations, such as difference methods and finite element methods. A mixed finite element method was established and the error estimate was carried out in [1], and a characteristics mixed finite element method was given in [2].

The collocation methods that meet constraint condition of interpolation have been developed since the 1970s. The methods satisfy the differential equation and the boundary conditions at collocation points by the piecewise polynomial approximation. Because the collocation method is easy to get approximation equations, does not need to compute numerical integration and has high-order accuracy, it is widely used in mathematical physics and engineering problems. For example, the collocation method in quasilinear parabolic equations was used in [3]. The collocation method in heat conduction equations was considered in [4].

Consider the linear sobolev equation with constant coefficients

$$
\left\{\begin{array}{l}
\mathrm{u}_{\mathrm{t}}=\nabla\left(\mathrm{a} \nabla \mathrm{u}_{\mathrm{t}}+\mathrm{b} \nabla \mathrm{u}\right)+\mathrm{f}(\mathrm{x}, \mathrm{y}),(\mathrm{x}, \mathrm{y}) \in \mathrm{I}, \mathrm{t} \in(0, \mathrm{~T}], \\
\left.\mathrm{u}\right|_{\partial \mathrm{I}}=0, \mathrm{x} \in \partial \mathrm{I}, \mathrm{t} \in[0, \mathrm{~T}] \\
\left.\mathrm{u}\right|_{\mathrm{t}=0}=\mathrm{u}_{0}(\mathrm{x}, \mathrm{y}),(\mathrm{x}, \mathrm{y}) \in \mathrm{I},
\end{array}\right.
$$

where $\mathrm{u}_{\mathrm{t}}$ denotes the time derivative of the function $\mathrm{u}, \nabla \mathrm{u}$ denotes the gradient of the function $\mathrm{u}$. And $\mathrm{a}, \mathrm{b}$ are known constants, $\mathrm{I}=[0,1] \times[0,1], \partial \mathrm{I}$ is the boundary of $\mathrm{I}$.

\section{The Orthogonal Collocation Method}

The domain $\mathrm{I}$ is dissected into equally spaced rectangular grid by grid points $\left(\mathrm{x}_{\mathrm{i}}, \mathrm{y}_{\mathrm{j}}\right), \mathrm{i}=0,1, \ldots, \mathrm{M}$; $\mathrm{j}=0,1, \ldots, \mathrm{N}$ and $0=\mathrm{x}_{0}<\mathrm{x}_{1}<\ldots<\mathrm{x}_{\mathrm{M}}<1 ; 0=\mathrm{y}_{0}<\mathrm{y}_{1}<\ldots<\mathrm{y}_{\mathrm{N}}<1$. Introduce the following notations:

$$
\begin{aligned}
& \mathrm{I}_{\mathrm{ij}}=\left[\mathrm{x}_{\mathrm{i}-1}, \mathrm{x}_{\mathrm{i}}\right] \times\left[\mathrm{y}_{\mathrm{j}-1}, \mathrm{y}_{\mathrm{j}}\right], \\
& \mathrm{h}_{\mathrm{x}}=\mathrm{x}_{\mathrm{i}}-\mathrm{x}_{\mathrm{i}-1}, \mathrm{~h}_{\mathrm{y}}=\mathrm{y}_{\mathrm{j}}-\mathrm{y}_{\mathrm{j}-1}, \mathrm{~h}=\max \left\{\mathrm{h}_{\mathrm{x}}, \mathrm{h}_{\mathrm{y}}\right\}, \\
& \mathrm{H}_{3}=\left\{\mathrm{v}=\mathrm{v}(\mathrm{x}, \mathrm{y}) \in \mathrm{C}^{1}(\mathrm{I})|\mathrm{v}|_{\mathrm{I}_{\mathrm{ij}}} \text { is a bi - cubic polynomial }, \mathrm{i}=1, \ldots, \mathrm{M}, \mathrm{j}=1,2, \ldots, \mathrm{N}\right\}, \\
& \mathrm{H}_{3}^{0}=\left\{\mathrm{v}=\mathrm{v}(\mathrm{x}) \in \mathrm{H}_{3}|\mathrm{v}|_{\partial \mathrm{I}}=0\right\} .
\end{aligned}
$$

We take four Gauss points as collocation points in $\mathrm{I}_{\mathrm{ij}}$ :

$$
\mathrm{x}_{\mathrm{ik}}=\mathrm{x}_{\mathrm{i}-1}+\mathrm{h}_{\mathrm{x}} \theta_{\mathrm{k}}, \mathrm{k}=1,2, \mathrm{y}_{\mathrm{jl}}=\mathrm{y}_{\mathrm{j}-1}+\mathrm{h}_{\mathrm{y}} \theta_{\mathrm{l}}, \mathrm{k}=1,2,1=1,2,
$$

where

$$
\theta_{1}=(3-\sqrt{3}) / 6, \theta_{2}=(3+\sqrt{3}) / 6
$$


Then we introduce a intermediate variable $\mathrm{q}=\mathrm{a} \nabla \mathrm{u}_{\mathrm{t}}+\mathrm{b} \nabla \mathrm{u}$. So we can building our orthogonal collocation method as follows:

Seeking for $(\mathrm{U}, \mathrm{Q}):[0, \mathrm{~T}] \rightarrow \mathrm{H}_{3} \times \mathrm{H}_{3}$, such that

$$
\begin{aligned}
& \left\{U_{t}-\nabla Q-f\right\}\left(x_{i k}, y_{j 1}, t\right)=0 ; \\
& \left\{Q-a \nabla U_{t}-b \nabla U\right\}\left(x_{i k}, y_{j 1}, t\right)=0 ; i=1, \ldots, M ; j=1, \ldots, N ; t \in(0, T] ; k, 1=1,2 ; \\
& \left\{\begin{array}{l}
\left.U\right|_{\partial I}=0, t \in[0, T] \\
\left.U\right|_{t=0}-u_{0}(x) \text { is sufficient ly small. }
\end{array}\right.
\end{aligned}
$$

\section{A Weighted Garlerkin Formulation}

We shall introduce some notation defined on $\mathrm{I}$ and $\mathrm{I}_{\mathrm{ij}}$ as follows:

$$
\begin{aligned}
& \langle\mathrm{u}, \mathrm{v}\rangle=\sum_{\mathrm{i}=1}^{\mathrm{M}} \sum_{\mathrm{j}=1}^{\mathrm{N}}\langle\mathrm{u}, \mathrm{v}\rangle_{\mathrm{ij}}=\sum_{\mathrm{i}=1}^{\mathrm{M}} \sum_{\mathrm{j}=1}^{\mathrm{N}} \frac{\mathrm{h}_{\mathrm{x}} \mathrm{h}_{\mathrm{y}}}{4} \sum_{\mathrm{k}, \mathrm{l}=1}^{2} \mathrm{uv}\left(\mathrm{x}_{\mathrm{ik}}, \mathrm{y}_{\mathrm{jl}}\right), \\
& \langle\mathrm{u}, \mathrm{v}\rangle_{\mathrm{x}}=\sum_{\mathrm{i}=1}^{\mathrm{M}}\langle\mathrm{u}, \mathrm{v}\rangle_{\mathrm{ix}}=\sum_{\mathrm{l}=1}^{\mathrm{M}} \frac{\mathrm{h}_{\mathrm{x}}}{2} \sum_{\mathrm{k}=1}^{2} \mathrm{uv}\left(\mathrm{x}_{\mathrm{ik}}, \mathrm{y}, \mathrm{t}\right), \\
& \langle\mathrm{u}, \mathrm{v}\rangle_{\mathrm{y}}=\sum_{\mathrm{j}=1}^{\mathrm{N}}\langle\mathrm{u}, \mathrm{v}\rangle_{\mathrm{jy}}=\sum_{\mathrm{l}=1}^{\mathrm{N}} \frac{\mathrm{h}_{\mathrm{y}}}{2} \sum_{\mathrm{l}=1}^{2} \mathrm{uv}\left(\mathrm{x}, \mathrm{y}_{\mathrm{jl}}, \mathrm{t}\right), \\
& \mid\|\| \mathrm{u}\left\|_{\mathrm{i}}^{2}=\langle\mathrm{u}, \mathrm{u}\rangle_{\mathrm{i}},\right\|\|\mathrm{u}\|^{2}=\langle\mathrm{u}, \mathrm{u}\rangle .
\end{aligned}
$$

Let $\mathrm{U}$ be the solution of (2). We can get the semi-discrete Galerkin method:

$$
\left\{\begin{array}{l}
\left\langle\mathrm{U}_{\mathrm{t}}, \mathrm{z}_{1}\right\rangle+\left\langle\mathrm{Q}, \nabla \mathrm{z}_{1}\right\rangle=\left\langle\mathrm{f}, \mathrm{z}_{1}\right\rangle, \\
\left\langle\mathrm{Q}, \mathrm{z}_{2}\right\rangle-\left\langle\mathrm{a} \nabla \mathrm{U}_{\mathrm{t}}+\mathrm{b} \nabla \mathrm{U}, \mathrm{z}_{2}\right\rangle=0 .
\end{array}\right.
$$

From the definition of $\langle\cdot$,$\rangle , the solutions of (2) and (3) are equivalent.$

Let $\left\{\zeta_{1}: l=1,2, \ldots, 4 M N\right\}=\left\{\left(x_{i k}, y_{j l}\right), i=1,2, \ldots, M, j=1,2, \ldots, N, k, l=1,2\right\}$ and $\left\{Z_{i}\right\}_{4 M N}$ is a group base of $\mathrm{H}_{3}^{0}$. So any $\mathrm{U}(\mathrm{x}, \mathrm{y})$ in $\mathrm{H}_{3}^{0}$ can expressed as follows

$$
\mathrm{U}(\mathrm{x}, \mathrm{y})=\sum_{\mathrm{i}=1}^{4 \mathrm{MN}} \beta_{\mathrm{i}}(\mathrm{t}) \mathrm{Z}_{\mathrm{i}}(\mathrm{x}, \mathrm{y})
$$

Then relations given by (2) and (3) can now be written as

$$
\mathrm{F} \beta_{\mathrm{t}}+\mathrm{G} \beta=\mathrm{R}, \mathrm{C} \beta_{\mathrm{t}}+\mathrm{A} \beta=\mathrm{S}
$$

where

$$
\begin{aligned}
& \mathrm{F}=\left(\mathrm{F}_{\mathrm{ij}}\right)_{4 \mathrm{MN} \times 4 \mathrm{MN}}, \mathrm{F}_{\mathrm{ij}}=\mathrm{Z}_{\mathrm{j}}\left(\zeta_{\mathrm{i}}\right)-\mathrm{a} \Delta \mathrm{Z}_{\mathrm{j}}\left(\zeta_{\mathrm{i}}\right) ; \mathrm{G}=\left(\mathrm{G}_{\mathrm{ij}}\right)_{4 \mathrm{MN} \times 4 \mathrm{MN}}, \mathrm{G}_{\mathrm{ij}}=-\mathrm{b} \Delta \mathrm{Z}_{\mathrm{j}}\left(\zeta_{\mathrm{i}}\right) ; \\
& \mathrm{C}=\left(\mathrm{C}_{\mathrm{ij}}\right)_{4 \mathrm{MN} \times 4 \mathrm{MN}}, \mathrm{C}_{\mathrm{ij}}=\left\langle\mathrm{Z}_{\mathrm{j}}-\mathrm{a} \Delta \mathrm{Z}_{\mathrm{j}}, \mathrm{Z}_{\mathrm{i}}\right\rangle ; \mathrm{A}=\left(\mathrm{A}_{\mathrm{ij}}\right)_{4 \mathrm{MN} \times 4 \mathrm{MN}}, \mathrm{A}_{\mathrm{ij}}=-\mathrm{b}\left\langle\Delta \mathrm{Z}_{\mathrm{j}}, \mathrm{Z}_{\mathrm{i}}\right\rangle ; \\
& \mathrm{R}=\left(\mathrm{R}_{\mathrm{i}}\right)_{4 \mathrm{MN}}, \mathrm{R}_{\mathrm{i}}=\mathrm{f}\left(\zeta_{\mathrm{i}}\right) ; \mathrm{S}=\left(\mathrm{S}_{\mathrm{i}}\right), \mathrm{S}_{\mathrm{i}}=\left\langle\mathrm{f}, \mathrm{Z}_{\mathrm{i}}\right\rangle .
\end{aligned}
$$

We notice that the $\tau$ satisfied $F \tau=0$ would also be the solution of $C \tau=0$. If $C$ is nonsingular, $F$ is also surely nonsingular. To achieve our conclusion, we just need that $\mathrm{C}$ is nonsingular. So we can prove it by counterevidence as follows: $\mathrm{C} \tau=0$.

Assume that $\mathrm{C}$ is singular. So we can find a $4 \mathrm{MN}$-dimensional nonzero vector $\tau$ which makes Let

$$
\mathrm{M}(\mathrm{x}, \mathrm{y})=\sum_{\mathrm{i}=1}^{4 \mathrm{MN}} \tau_{\mathrm{i}} \mathrm{Z}_{\mathrm{i}}(\mathrm{x}, \mathrm{y}), \tau=\left(\tau_{\mathrm{i}}\right)_{4 \mathrm{MN}}
$$


we can get $\langle M, M\rangle=0$ from $C \tau=0$. Furthermore we can get $M\left(\zeta_{1}\right)=0,1=1,2, \ldots, 4 M N$.From lemma 1.2 of [4], there is $\mathrm{M}(\mathrm{x}, \mathrm{y})=0$. So $\tau=0$ and it conflicts to the assumption. Then $\mathrm{C}$ is nonsingular and the solution of (2) and (3) are equivalent.

\section{Convergence Analysis}

Then we can define a map $(\mathrm{W}, \mathrm{V}):[0, \mathrm{~T}] \rightarrow \mathrm{H}_{3} \times \mathrm{H}_{3}$ such that ${ }^{[1]}$

$$
\begin{aligned}
& \langle\nabla(\mathrm{q}-\mathrm{V}), \mathrm{z}\rangle=0, \forall \mathrm{z} \in \mathrm{H}_{3}, \\
& \left\langle\mathrm{a} \nabla\left(\mathrm{u}_{\mathrm{t}}-\mathrm{W}_{\mathrm{t}}\right)+\mathrm{b} \nabla(\mathrm{u}-\mathrm{W}), \nabla \mathrm{z}\right\rangle=0, \forall \mathrm{z} \in \mathrm{H}_{3}, \\
& \left\langle\mathrm{u}_{0}-\mathrm{W}(0), \mathrm{z}\right\rangle, \forall \mathrm{z} \in \mathrm{H}_{3} .
\end{aligned}
$$

We can get the following convergence conclusion for the above numerical Scheme.

Theorem: When $\mathrm{u} \in \mathrm{L}^{\infty}\left(0, \mathrm{~T} ; \mathrm{H}^{6}(\mathrm{I})\right) \cap \mathrm{L}^{2}\left(0, \mathrm{~T} ; \mathrm{H}^{6}(\mathrm{I})\right), \mathrm{u}_{\mathrm{t}} \in \mathrm{L}^{2}\left(0, \mathrm{~T} ; \mathrm{H}^{6}(\mathrm{I})\right)$, then there exists a constant $K$ such that, for $h$ sufficiently small

$$
\|\mathrm{u}-\mathrm{U}\| \leq \mathrm{Kh}^{4}, \quad\|\mathrm{q}-\mathrm{Q}\| \leq \mathrm{Kh}^{4},
$$

Proof: Let $\mathrm{v}=\mathrm{W}-\mathrm{U}, \eta=\mathrm{u}-\mathrm{W}, \mathrm{w}=\mathrm{V}-\mathrm{Q}, \xi=\mathrm{q}-\mathrm{V}$, so $\mathrm{u}-\mathrm{U}=\mathrm{v}+\eta, \mathrm{q}-\mathrm{Q}=\mathrm{w}+\xi$. The following conclusion in [4] will be needed:

$$
\begin{aligned}
& \|\eta\| \leq \operatorname{ch}^{4}\left(\sum_{i, j}\left\|u^{(4)}\right\|^{2}\right)^{1 / 2},\left\|\eta_{t}\right\| \leq \operatorname{ch}^{4}\left(\sum_{i, j}\left\|u_{t}^{(4)}\right\|^{2}\right)^{1 / 2}, \\
& \|\xi\| \leq \operatorname{ch}^{4}\left(\sum_{i, j}\left\|q^{(4)}\right\|^{2}\right)^{1 / 2},\left\|\xi_{t}\right\| \leq \operatorname{ch}^{4}\left(\sum_{i, j}\left\|q_{t}^{(4)}\right\|^{2}\right)^{1 / 2},
\end{aligned}
$$

where $\mathrm{c}$ is a positive constant and $\mathrm{u}$ is sufficiently smooth.

So we can get the error equations:

$$
\left\{\begin{array}{l}
\left\langle\eta_{\mathrm{t}}, \mathrm{z}_{1}\right\rangle+\left\langle\mathrm{v}_{\mathrm{t}}, \mathrm{z}_{1}\right\rangle+\left\langle\xi, \nabla \mathrm{z}_{1}\right\rangle+\left\langle\mathrm{w}, \nabla \mathrm{z}_{1}\right\rangle=0 \\
\left\langle\xi, \mathrm{z}_{2}\right\rangle+\left\langle\mathrm{w}, \mathrm{z}_{2}\right\rangle-\left\langle\mathrm{a} \nabla \mathrm{v}_{\mathrm{t}}+\mathrm{b} \nabla \mathrm{v}, \mathrm{z}_{2}\right\rangle=0 .
\end{array}\right.
$$

Firstly, the choice $\mathrm{z}_{1=\mathrm{V}}, \mathrm{z}_{2}=\nabla \mathrm{v}$ leads to the equations

$$
\left\{\begin{array}{l}
\left\langle\eta_{\mathrm{t}}, \mathrm{v}\right\rangle+\left\langle\mathrm{v}_{\mathrm{t}}, \mathrm{v}\right\rangle+\langle\xi, \nabla \mathrm{v}\rangle+\langle\mathrm{w}, \nabla \mathrm{v}\rangle=0, \\
\langle\xi, \nabla \mathrm{v}\rangle+\langle\mathrm{w}, \nabla \mathrm{v}\rangle-\left\langle\mathrm{a} \nabla \mathrm{v}_{\mathrm{t}}+\mathrm{b} \nabla \mathrm{v}, \nabla \mathrm{v}\right\rangle=0 .
\end{array}\right.
$$

The first equation in (5) minus the second one. We can see

$$
\left\langle\mathrm{a} \nabla \mathrm{v}_{\mathrm{t}}, \nabla \mathrm{v}\right\rangle+\left\langle\mathrm{v}_{\mathrm{t}}, \mathrm{v}\right\rangle=-\langle\mathrm{b} \nabla \mathrm{v}, \nabla \mathrm{v}\rangle-\left\langle\eta_{\mathrm{t}}, \mathrm{v}\right\rangle
$$

Add Cauchy inequality and the equation

$$
\left\langle\nabla \mathrm{v}_{\mathrm{t}}, \nabla \mathrm{v}\right\rangle=\frac{1}{2} \frac{\mathrm{d}}{\mathrm{dt}}\|\nabla \mathrm{v}\|^{2}
$$

we can obtain

$$
\begin{gathered}
\left.\frac{\mathrm{a}}{2} \frac{\mathrm{d}}{\mathrm{dt}}\|\nabla \mathrm{v}\|\right|^{2}+\frac{1}{2} \frac{\mathrm{d}}{\mathrm{dt}}\|\| \mathrm{v} \|\left.\right|^{2}=\mathrm{a}\left\langle\nabla \mathrm{v}_{\mathrm{t}}, \nabla \mathrm{v}\right\rangle+\left\langle\mathrm{v}_{\mathrm{t}}, \mathrm{v}\right\rangle+\frac{1}{2}\langle\mathrm{v}, \mathrm{v}\rangle, \\
\frac{\mathrm{d}}{\mathrm{dt}}\|\mid \nabla \mathrm{v}\|\left\|^{2}+\frac{\mathrm{d}}{\mathrm{dt}}\right\|\|\mathrm{v}\|^{2} \leq \mathrm{K}\left(\langle\nabla \mathrm{v}, \nabla \mathrm{v}\rangle+\left\langle\eta_{\mathrm{t}}, \mathrm{v}\right\rangle+\langle\mathrm{v}, \mathrm{v}\rangle\right) \\
\leq \mathrm{K}\left(\|\mid \nabla \mathrm{v}\|\left\|^{2}+\right\|\left\|\eta_{\mathrm{t}}\right\|^{2}+\|\mathrm{v}\|^{2}\right) .
\end{gathered}
$$

where $\mathrm{K}$ is a positive constant. Integrating the above equation, we get

$$
\left\|\left|\nabla \mathrm{v}\left\|\left.\right|^{2}+\right\| \mathrm{v}\right|\right\|^{2} \leq \mathrm{K} \int_{0}^{\mathrm{T}}\left(\left\|\left|\nabla \mathrm{v}\left\|\left.\right|^{2}+\right\|\right| \eta_{\mathrm{t}}\left|\left\|^{2}+\right\| \mathrm{v} \|\right|^{2}\right) \mathrm{ds} .\right.
$$


It follows from the Gronwall lemma that

$$
\|\nabla \mathrm{v}\|^{2}+\|\mathrm{v}\|^{2} \leq \mathrm{K} \int_{0}^{\mathrm{T}}\left(\|\| \eta_{\mathrm{t}} \|^{2}\right) \mathrm{ds}
$$

Secondly the choice $\mathrm{z}_{1=} \mathrm{v}_{\mathrm{t}}, \mathrm{z}_{2}=\nabla \mathrm{v}_{\mathrm{t}}$ leads to the equations

$$
\left\{\begin{array}{l}
\left\langle\eta_{\mathrm{t}}, \mathrm{v}_{\mathrm{t}}\right\rangle+\left\langle\mathrm{v}_{\mathrm{t}}, \mathrm{v}_{\mathrm{t}}\right\rangle+\left\langle\xi, \nabla \mathrm{v}_{\mathrm{t}}\right\rangle+\left\langle\mathrm{w}, \nabla \mathrm{v}_{\mathrm{t}}\right\rangle=0, \\
\left\langle\xi, \nabla \mathrm{v}_{\mathrm{t}}\right\rangle+\left\langle\mathrm{w}, \nabla \mathrm{v}_{\mathrm{t}}\right\rangle-\left\langle a \mathrm{v}_{\mathrm{t}}+\mathrm{b} \nabla \mathrm{v}, \nabla \mathrm{v}_{\mathrm{t}}\right\rangle=0 .
\end{array}\right.
$$

The first equation in (7) minus the second one. We can see:

$$
\left\langle a v_{\mathrm{t}}, \nabla \mathrm{v}_{\mathrm{t}}\right\rangle+\left\langle\mathrm{v}_{\mathrm{t}}, \mathrm{v}_{\mathrm{t}}\right\rangle=-\left\langle\mathrm{b} \nabla \mathrm{v}, \nabla \mathrm{v}_{\mathrm{t}}\right\rangle-\left\langle\eta_{\mathrm{t}}, \mathrm{v}_{\mathrm{t}}\right\rangle .
$$

Add Cauchy inequality to obtain

$$
\begin{aligned}
\left\|\left|\nabla \mathrm{v}_{\mathrm{t}}\right|\right\|^{2}+\left\|\left|\mathrm{v}_{\mathrm{t}}\right|\right\|^{2} & \leq \mathrm{K}\left(\|\| \eta_{\mathrm{t}}\left|\left\|^{2}+\right\|\right|\|\mathrm{v}\|^{2}\right) \\
& \leq \mathrm{K}\left(\|\| \eta_{\mathrm{t}}\left\|^{2}+\int_{0}^{\mathrm{T}}\right\|\left|\eta_{\mathrm{t}} \|\right|^{2} \mathrm{ds}\right) .
\end{aligned}
$$

Then the choice $\mathrm{z}_{2}=\mathrm{w}$ leads to the equation

$$
\langle\xi, \mathrm{w}\rangle+\langle\mathrm{w}, \mathrm{w}\rangle=\left\langle\mathrm{a} \nabla \mathrm{v}_{\mathrm{t}}+\mathrm{b} \nabla \mathrm{v}, \mathrm{w}\right\rangle
$$

It follows that

$$
\begin{aligned}
\|\| \mathrm{w}\|\|^{2}=\left\langle\mathrm{a} \nabla \mathrm{v}_{\mathrm{t}}+\mathrm{b} \nabla \mathrm{v}, \mathrm{w}\right\rangle-\langle\xi, \mathrm{w}\rangle & \leq \mathrm{K}\left(\|\| \xi\left\|^{2}+\right\| \nabla \mathrm{v}_{\mathrm{t}}\left\|^{2}+\right\| \mid\|\mathrm{v}\| \|^{2}\right) \\
& \leq \mathrm{K}\left(\|\xi \xi\|^{2}+\|\| \eta_{\mathrm{t}}\left|\left\|^{2}+\int_{0}^{\mathrm{T}}\right\|\left\|\eta_{\mathrm{t}} \mid\right\|^{2} \mathrm{ds}\right) .\right.
\end{aligned}
$$

Then (2,2) of [5] implies that $\forall \mathrm{f} \in \mathrm{H}_{3},\|\mathrm{f}\| \leq\|\mathrm{f}\| \mid \leq \mathrm{K}\|\mathrm{f}\|$. So combine with(4), (6),(8) and (9), so we can obtain

$$
\begin{aligned}
& \|\mathrm{u}-\mathrm{U}\|^{2} \leq\|\eta\|^{2}+\|\mathrm{v}\|^{2} \leq\|\eta\|^{2}+\|\mathrm{v}\|^{2} \leq\|\eta\|^{2}+\mathrm{K} \int_{0}^{\mathrm{T}}\left\|\eta_{\mathrm{t}}\right\|^{2} \mathrm{ds} \leq \mathrm{K}_{1} \mathrm{~h}^{8}, \\
& \|\mathrm{q}-\mathrm{Q}\|^{2} \leq\|\xi\|^{2}+\|\mathrm{w}\|^{2} \leq\|\xi\|^{2}+\|\mathrm{w}\|^{2} \leq\|\xi\|^{2}+\mathrm{K}\left(\|\xi\|^{2}+\left\|\eta_{\mathrm{t}}\right\|^{2}+\int_{0}^{\mathrm{T}}\left\|\eta_{\mathrm{t}}\right\|^{2} \mathrm{ds}\right) \leq \mathrm{K}_{1} \mathrm{~h}^{8},
\end{aligned}
$$

for $h$ sufficiently small, and the proof is complete.

\section{Acknowledgements}

We thank the fund "Basic Subjects Fund of China University of Petroleum (Beijing) (2462015YQ0604)".

\section{References}

[1] Jiang Ziwen, Chen Huanzhen, Error estimates for mixed finite element methods for sobolev equation, Northeast Math J,17(3) (2001) 301-314.

[2] Zhao Peixin, Chen Huanzhen, A characteristics mixed finite element method for sobolev equations ,Mathematica Applicata, 16(4) (2003) 50-59.

[3] Jim Douglas,Jr.,Todd Dupon, A finite element collocation method for quasilinear parabolic equations, Mathematics of Computation, 27 (1973) 17-28.

[4] Tongchao $\mathrm{Lu}$, The finite element collocation method of Initial and boundary value problem about two-dimensional heat conduction equation,Chinese Shandong University J,29 (1994) 266-272.

[5] R. I. Fernandes, G. Fairweather, Analysis of alternating direction collocation methods for parabolic and hyperbolic problems in two space variables, Numer Methods Partial Differential Eq, 9 (1993) 191-211. 